\title{
Note on Translations and Quotations
}

All translations except those from Greek texts are my own. In quoting from foreign languages and from Renaissance English texts, I have normalized spelling, changing i's to j's and u's to v's (and vice versa), when necessary. I have also expanded scribal abbreviations and removed accent marks in the Latin. 

The Emperor of Men's Minds 
